\title{
Energy spectrum of ultra-high energy cosmic rays according to surface detectors of Yakutsk EAS array
}

\author{
A. V. Sabourov*, A. V. Glushkov, M. I. Pravdin, Yu. A. Egorov, A. A. Ivanov, \\ S. P. Knurenko, A. D. Krasilnikov, I. T. Makarov, V. P. Mokhnachevskaya, \\ A. A. Mikhailov, S. V. Matarkin, Z. E. Petrov, I. S. Petrov, I. Ye. Sleptsov, \\ G. G. Struchkov, L. V. Timofeev \\ $Y$ Y. G. Shafer Institute of cosmophysical research and aeronomy SB RAS \\ E-mail: tema@ikfia.ysn.ru
}

\begin{abstract}
We present the results of the analysis of cosmic rays spectrum in energy domain above $10^{17} \mathrm{eV}$. The analysis covers extensive air showers registered over the course of continuous observations from 1974 to 2017. A new refined relation was applied to reconstruct primary energy in individual events. It was derived from the lateral distribution of responses of surface and underground detectors of the Yakutsk EAS array obtained in air shower simulations with four hadron interaction models - QGSJet01D, QGSJet-II-04, SIBYLL-2.1 and EPOS-LHC. The resulting new energy estimation is 1.4 times lower than the values obtained earlier with calorimetric method.
\end{abstract}

35th International Cosmic Ray Conference - ICRC2017

10-20 July, 2017

Bexco, Busan, Korea

${ }^{*}$ Speaker. 


\section{Introduction}

Energy spectrum of ultra-high energy cosmic rays (UHECR) - cosmic rays (CR) with energy above $\sim 10^{17} \mathrm{eV}-$ is one of the keys to understanding the nature of these particles. Experimental results obtained on different extensive air shower (EAS) arrays [1-8] closely reproduce the shape of the spectrum but their intensities differ from each other by factor of almost 2 [9]. Such situation can be mainly explained by the fact that CR observation method based on EAS registration is indirect and each experiment uses its own technique to reconstruct the energy of primary particles $\left(E_{0}\right)$. Thus, on cannot do without theoretical notion on EAS development.

The Yakutsk EAS array is the world's oldest UHECR experiment - it's been continuously operating since 1974. It stands out from the other large arrays by the ability to simultaneously measure several shower components, including charged particles, electromagnetic component, muons (with $1 \mathrm{GeV}$ threshold) and Cherenkov light emission. The Cherenkov component contains $\sim 80 \%$ of primary energy dissipated by shower in the Earth's atmosphere and allows to estimate it with the use of calorimertic method [10-13]. Initially this method was utilized for interpretation of events described in [14] for energy $\sim 10^{15} \mathrm{eV}$. In Yakutsk experiment it is applied in energy range $\sim(1.0-100) \times 10^{17} \mathrm{eV}$ and zenith angles $\theta \leq 45^{\circ}$ :

$$
\begin{array}{r}
E_{0}=(4.1 \pm 1.4) \times 10^{17} \cdot \rho_{s, 600}\left(0^{\circ}\right)^{0.97 \pm 0.04}(\mathrm{eV}), \\
\rho_{s}\left(600,0^{\circ}\right)=\rho_{s, 600}(\theta) \cdot \exp (\sec \theta-1) \cdot \frac{1020}{\lambda}\left(\mathrm{m}^{-2}\right), \\
\lambda=400 \pm 45 \quad\left(\mathrm{~g} / \mathrm{cm}^{2}\right),
\end{array}
$$

where $\rho_{s, 600}(\theta)$ - particles density measured by surface scintillation detectors (SSD) at the distance $600 \mathrm{~m}$ from shower axis. Later, equations (1.1)-(1.3) were changed slightly:

$$
\begin{aligned}
E_{0} & =(4.8 \pm 1.6) \times 10^{17} \cdot \rho_{s, 600}\left(0^{\circ}\right)^{1.0 \pm 0.02}(\mathrm{eV}), \\
\lambda & =(450 \pm 44)+(32 \pm 15) \cdot \log _{10} \rho_{s, 600}\left(0^{\circ}\right)\left(\mathrm{g} / \mathrm{cm}^{2}\right)
\end{aligned}
$$

The intensity of CR spectrum derived from (1.4) was significantly higher than all the world data. In works $[15,16]$ we reconsidered the energy calibration of the array with the use of CORSIKA program [17] which is described further.

\section{Primary energy estimation}

We calculated the lateral distribution function (LDF) of the SSD response according to QGSJet01D [18], QGSJet-II-04 [19], SIBYLL-2.1 [20] and EPOS-LHC [21] hadron interaction models for primary protons and iron nuclei. FLUKA model [22] was chosen for treatment of of low energy hadron interactions. The details of the detector response estimation were outlined in $[15,16]$.

The dependence of the value $\log _{10}\left(\rho_{s, 600}\left(0^{\circ}\right) / E_{0}\right)$ obtained within the framework of QGSJet01D model is well described by the relation:

$$
E_{0}=(3.55 \pm 0.1) \times 10^{17} \cdot \rho_{s, 600}\left(0^{\circ}\right)^{1.02}(\mathrm{eV}) .
$$


Other models (QGSJet-II-04, EPOS-LHC and SIBYLL-2.1) gave the following estimations correspondingly:

$$
\begin{aligned}
& E_{0}=(3.19 \pm 0.1) \times 10^{17} \cdot \rho_{s, 600}(0)^{1.03}(\mathrm{eV}), \\
& E_{0}=(2.87 \pm 0.1) \times 10^{17} \cdot \rho_{s, 600}(0)^{1.03}(\mathrm{eV}), \\
& E_{0}=(3.72 \pm 0.1) \times 10^{17} \cdot \rho_{s, 600}(0)^{1.02}(\mathrm{eV})
\end{aligned}
$$

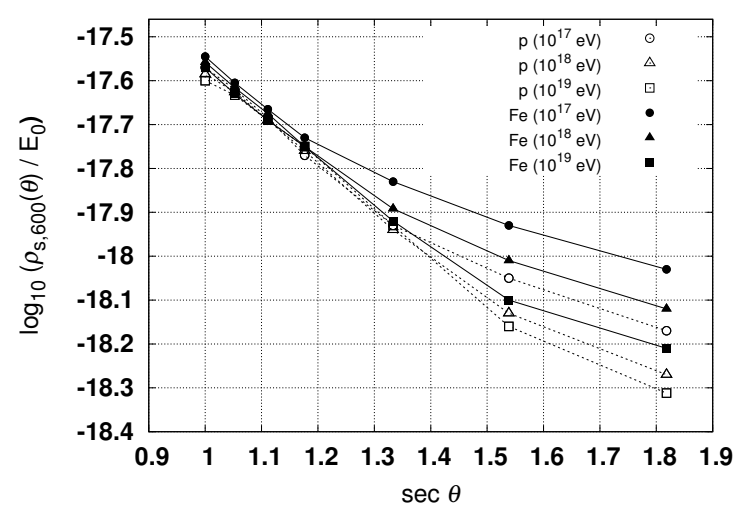

Figure 1: Zenith-angular dependences of $\log _{10}\left(\rho_{s, 600}(\theta) / E_{0}\right)$ according to QGSJet01Dpredictions for protons and iron nuclei with energy $E_{0}=10^{17}, 10^{18}$ and $10^{19} \mathrm{eV}$.

Zenith-angular dependences of $\log _{10}\left(\rho_{s, 600}(\theta) / E_{0}\right)$ according to QGSJet01D model are shown on Fig. 1. They correspond to changed $\lambda$ in (1.2) shown on Fig. 2. Dashed line on the plot represents the absorption range of mixed composition of primary nuclei according to our experimental data [23, 24], dotted line - empirical relation (1.5).

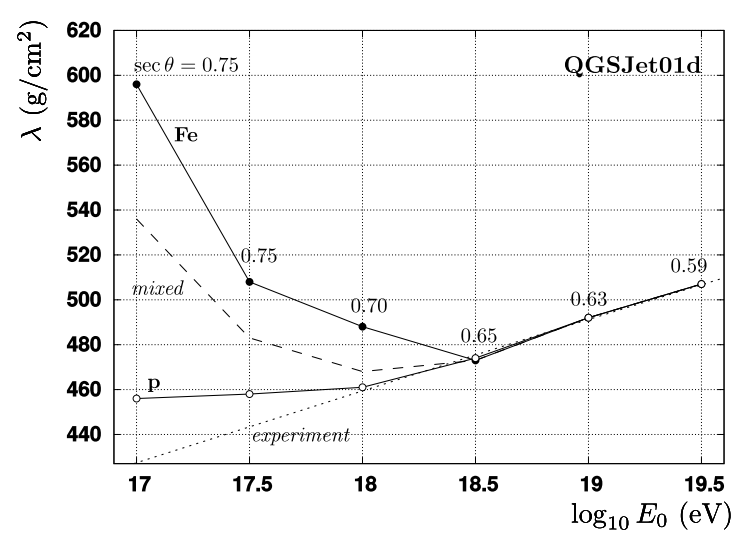

Figure 2: Values of absorption range in equation (1.2) used for recalculation of $\rho_{s, 600}(\theta)$ in inclined to vertical showers according to QGSJet01D model for primary protons, mixed composition and iron nuclei, shown as dependence of primary energy. Marginal allowed angles are denoted by numbers. Dotted line represents relation (1.5).

The calorimetric method was applied to the set of experimental data from [10,11]. The observables and main components constituting the primary energy are given in Tables 1 and 2 for energy $10^{18} \mathrm{eV}$ and $\cos \theta=0.95$. The row entitled "average $\mathrm{p}-\mathrm{Fe} "$ corresponds to values averaged over all 
models and compositions. The energy dissipated in the atmosphere by electromagnetic component equals to:

$$
E_{i}=E_{\gamma}+E_{\text {ion }},
$$

where $E_{\gamma}$ is energy of gamma-photons on observation level, $E_{\text {ion }}-$ summary ionization losses of all electrons and positrons. It is proportional to the total flux $F$ of Cherenkov radiation in the atmosphere:

$$
E_{i} \propto k \cdot F,
$$

where $k$ is the scaling factor: $k=k_{\gamma}+k_{\text {ion }}=\frac{E_{\gamma}+E_{\text {ion }}}{F}\left(\mathrm{eV} /\right.$ photon $\left.\mathrm{eV}^{-1}\right)$.

The flux $F$ is determined with respect to its attenuation by factor 1.15 due to Rayleigh scattering in clean atmosphere and degradation of the relative transparency in sampling events $[10,11]$ by factor 1.1. It is given for radiation interval $1 \mathrm{eV}$ :

$$
F=1.265 \cdot \frac{F_{\mathrm{obs}}}{\Delta \varepsilon},
$$

where $F_{\mathrm{obs}}$ is the flux measured in experiment with integral Cherenkov light detectors and $\Delta \varepsilon \simeq$ $2.58 \mathrm{eV}$.

The energy $E_{\mathrm{el}}$ is the fraction of primary energy carried by electrons and positrons to the observation level. It was estimated by integrating the differential energy deposit over the cascade curve $N_{e}(x)$ below the observation level $x_{\text {obs }}$ (see $\left.[15,16]\right)$.

Energy of muons was measured experimentally:

$$
E_{\mu} \simeq\left\langle E_{1 \mu}\right\rangle \cdot\left\langle N_{\mu}\left(x_{\mathrm{obs}}\right)\right\rangle,
$$

where $\left\langle E_{1 \mu}\right\rangle=10.6 \mathrm{GeV}$, which is the mean energy of single muon.

From the data given in Table 2, averaged over all models, the summary value $E_{i}+E_{\mathrm{el}}+E_{\mu}$ amounts to $\sim 93 \%$ from primary energy. The rest of it $(\Delta E)$ is not controlled by the array. It includes energy of neutrinos, energy transferred to nuclei in various reactions and ionization losses of muons and hadrons in the atmosphere. In $[10,11]$ this value was obtained from earlier calculations and is roughly consistent with predictions obtained with CORSIKA.

Summary values of all constituents are given in the rightmost column of the Table 2 . The value $E_{0}=1.173 \times 10^{18} \mathrm{eV}$ in the "experiment" column exceeds the mean value $E_{0}=0.997 \times 10^{18} \mathrm{eV}$ obtained in simulation by factor $\sim 1.177$. This difference is a result of overestimation of the scaling factor $k$, occurred in $[10,11]$, where it was determined as $k=3.7 \times 10^{4} \mathrm{eV} /$ photon $\mathrm{eV}^{-1}$, while simulation with CORSIKAgave $k=3.157 \times 10^{4} \mathrm{eV} /$ photon $\mathrm{eV}^{-1}$. The new estimation of primary energy obtained with the use of calorimetric method described above is given in the bottom row of the Table 2. The value $E_{0}=1.019 \times 10^{18} \mathrm{eV}$ was determined with corrected values $E_{i}=k \cdot F$, $E_{1 \mu}=10.3 \mathrm{GeV}$ and $\Delta E$. It is shown on Fig. 3 together with other data from [10] with black circles. White circles represent the data from [11] reprocessed with the revised values of $F$ and $E_{\text {ion }}$ with the account of the adjusted atmosphere transparency and with introduction of a new scaling factor $k$. Solid line represents the dependency:

$$
E_{0}=(3.76 \pm 0.3) \times 10^{17} \times \rho_{s, 600}(0)^{1.02 \pm 0.02}(\mathrm{eV})
$$




\begin{tabular}{lccccccc}
\hline model & $A$ & $\begin{array}{c}k_{\gamma}(\theta) \\
\times 10^{4} \\
\mathrm{eV}^{2}\end{array}$ & $\begin{array}{c}k_{\text {ion }}(\theta) \\
\times 10^{4} \\
\mathrm{eV}^{2}\end{array}$ & $\begin{array}{c}F(\theta) \\
\times 10^{13} \\
\mathrm{eV}^{-1}\end{array}$ & $\begin{array}{c}N_{s}(\theta) \\
\times 10^{8}\end{array}$ & $\begin{array}{c}\rho_{s, 600}(\theta) \\
\mathrm{m}^{-2}\end{array}$ & $\begin{array}{c}N_{\mu}(\theta) \\
\times 10^{6}\end{array}$ \\
\hline QGSJet01D & $\mathrm{p}$ & 0.341 & 2.846 & 2.104 & 2.178 & 2.312 & 5.000 \\
& $\mathrm{Fe}$ & 0.224 & 2.910 & 2.148 & 1.250 & 2.432 & 7.225 \\
QGSJet-II-04 & $\mathrm{p}$ & 0.364 & 2.816 & 2.070 & 2.296 & 2.438 & 5.582 \\
& $\mathrm{Fe}$ & 0.246 & 2.894 & 2.148 & 1.358 & 2.636 & 7.777 \\
SIBYLL-2.1 & $\mathrm{p}$ & 0.345 & 2.822 & 2.100 & 2.512 & 2.193 & 4.254 \\
& $\mathrm{Fe}$ & 0.224 & 2.910 & 2.228 & 1.384 & 2.249 & 4.930 \\
EPOS-LHC & $\mathrm{p}$ & 0.377 & 2.815 & 2.023 & 2.355 & 2.655 & 5.905 \\
& $\mathrm{Fe}$ & 0.230 & 2.894 & 2.133 & 1.419 & 2.917 & 8.180 \\
average & $\mathrm{p}$ & 0.357 & 2.825 & 2.074 & 2.335 & 2.400 & 5.185 \\
average & $\mathrm{Fe}$ & 0.231 & 2.902 & 2.164 & 1.353 & 2.558 & 7.028 \\
average & $\mathrm{p}-\mathrm{Fe}$ & 0.294 & 2.864 & 2.119 & 1.844 & 2.479 & 6.107 \\
experiment & & & 3.700 & 2.510 & 1.793 & 2.656 & 6.00 \\
\hline
\end{tabular}

Table 1: Observables of EAS with energy $10^{18} \mathrm{eV}$ and $\cos \theta=0.95$ initiated by different primary nuclei $(A)$ according to simulations and experiment $[10,11]$

\begin{tabular}{lccccccc}
\hline model & $\mathrm{A}$ & $\begin{array}{c}E_{\gamma} \\
\times 10^{17} \\
\mathrm{eV}\end{array}$ & $\begin{array}{c}E_{\text {ion }} \\
\times 10^{17} \\
\mathrm{eV}\end{array}$ & $\begin{array}{c}E_{\mathrm{el}} \\
\times 10^{17} \\
\mathrm{eV}\end{array}$ & $\begin{array}{c}E_{\mu} \\
\times 10^{17} \\
\mathrm{eV}\end{array}$ & $\begin{array}{c}\Delta E \\
\times 10^{17} \\
\mathrm{eV}\end{array}$ & $\begin{array}{c}E_{0} \\
\times 10^{17} \\
\mathrm{eV}\end{array}$ \\
\hline QGSJet01D & $\mathrm{p}$ & 0.806 & 6.620 & 1.469 & 0.517 & 0.565 & 9.978 \\
& $\mathrm{Fe}$ & 0.529 & 6.600 & 1.306 & 0.785 & 0.798 & 9.972 \\
QGSJet-II-04 & $\mathrm{p}$ & 0.859 & 6.476 & 1.474 & 0.547 & 0.624 & 9.980 \\
& $\mathrm{Fe}$ & 0.582 & 6.430 & 1.302 & 0.844 & 0.866 & 9.981 \\
SIBYLL-2.1 & $\mathrm{p}$ & 0.909 & 6.625 & 1.523 & 0.428 & 0.491 & 9.976 \\
& $\mathrm{Fe}$ & 0.528 & 6.679 & 1.340 & 0.702 & 0.716 & 9.965 \\
EPOS-LHC & $\mathrm{p}$ & 0.891 & 6.412 & 1.482 & 0.524 & 0.657 & 9.966 \\
& $\mathrm{Fe}$ & 0.543 & 6.415 & 1.305 & 0.794 & 0.898 & 9.955 \\
average & $\mathrm{p}$ & 0.866 & 6.533 & 1.487 & 0.504 & 0.584 & 9.974 \\
average & $\mathrm{Fe}$ & 0.546 & 6.531 & 1.313 & 0.781 & 0.820 & 9.968 \\
average & $\mathrm{p}-\mathrm{Fe}$ & 0.706 & 6.532 & 1.400 & 0.643 & 0.702 & 9.970 \\
experiment & - & - & 9.287 & 0.947 & 0.636 & 0.860 & 11.73 \\
refinement & - & - & 7.926 & 0.947 & 0.618 & 0.702 & 10.19 \\
\hline
\end{tabular}

Table 2: Energy balance in EAS with $E_{0}=10^{18} \mathrm{eV}$ and $\cos \theta=0.95$ from different primary nuclei $(A)$ according to simulations and experiment $[10,11]$ 


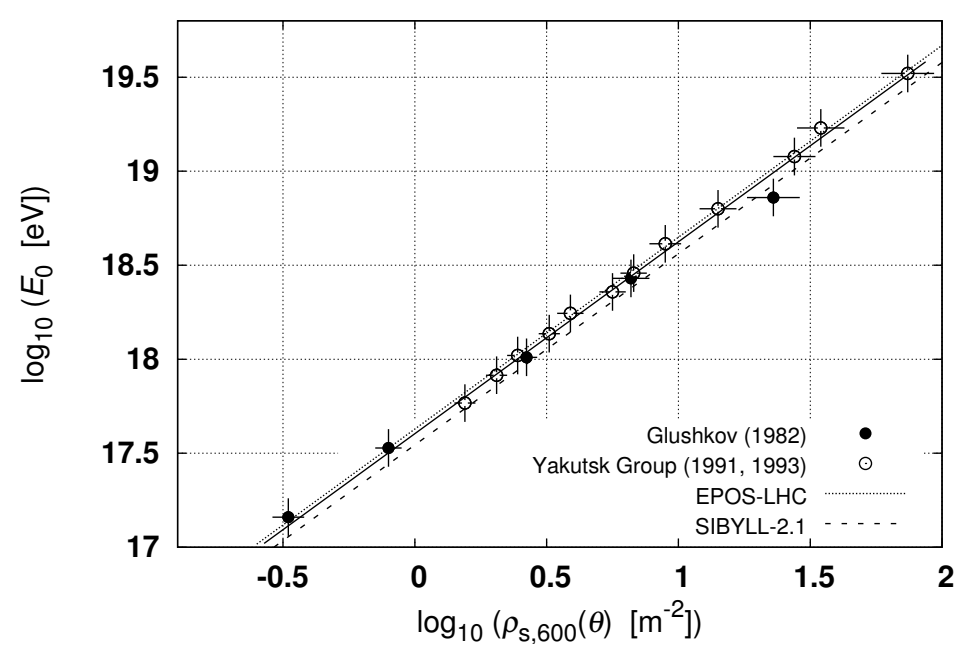

Figure 3: The $\rho_{s, 600}(\theta)$ dependence of $E_{0}$ in showers with $\langle\cos \theta\rangle=0.95$ according to data from [10] (dark circles) and [11] (white circles) obtained with new calorimetry. Solid line represents the best fit to all data. Dashed and dotted lines represent relations (2.3) and (2.4) correspondingly for given zenith angle.

which describes all the experimental data when $\rho_{s, 600}\left(18.2^{\circ}\right)$ is converted to vertical with the use of relation (1.2) with absorption ranges $\lambda$ which is shown on Fig. 2 with dashed line (i.e. for mixed CR composition). Dotted and dashed lines on Fig. 3 reflect the relations (2.3) and (2.4) which signify limits of the interval containing predictions of all the abovementioned models. The closest to experiment are QGSJet01D and SIBYLL-2.1.

\section{Primary energy spectrum}

For this analysis we have evaluated more than $10^{6}$ shower events registered during the period of continuous operation of Yakutsk array, lasting from 1974 to 2017. The spectrum was obtained with the use of a technique described in [25]. Energy in individual showers was reconstructed according to the refined calorimetric formula (2.9) which is almost model-independent and is based on the agreeing results (see Table 2). Absorption ranges were obtained with the use of equation (1.3) for real mixed composition [23,24]. The resulting spectrum is shown on Fig. 4 with dark circles. Light circles represent spectrum from [26] obtained from measurements of Cherenkov light emission. Other symbols represent the data from Akeno (1984, 1992) [27, 28], AGASA [29], Tunka-133 [30], IceTop [31], HiRes I [6], HiRes II [32], Telescope Array (TA) [7] and from The Pierre Auger Observatory (PAO) [8].

Our spectrum and spectra from Akeno-AGASA [27-29] agree with each other in all energy range. This is possibly due to similarities in scintillation detectors and analysis techniques utilized in these experiments. There is also a good agreement between Yakutsk experiment, Tunka-133 and IceTop at $E_{0} \sim 10^{17} \mathrm{eV}$. On the other hand, there is significant difference from HiRes and PAO\&TA at $E_{0}>10^{18} \mathrm{eV}$ which might be caused by technical features of these arrays.

\section{Conclusion}

With the use of modern hadron interaction models we have managed to estimate the response 


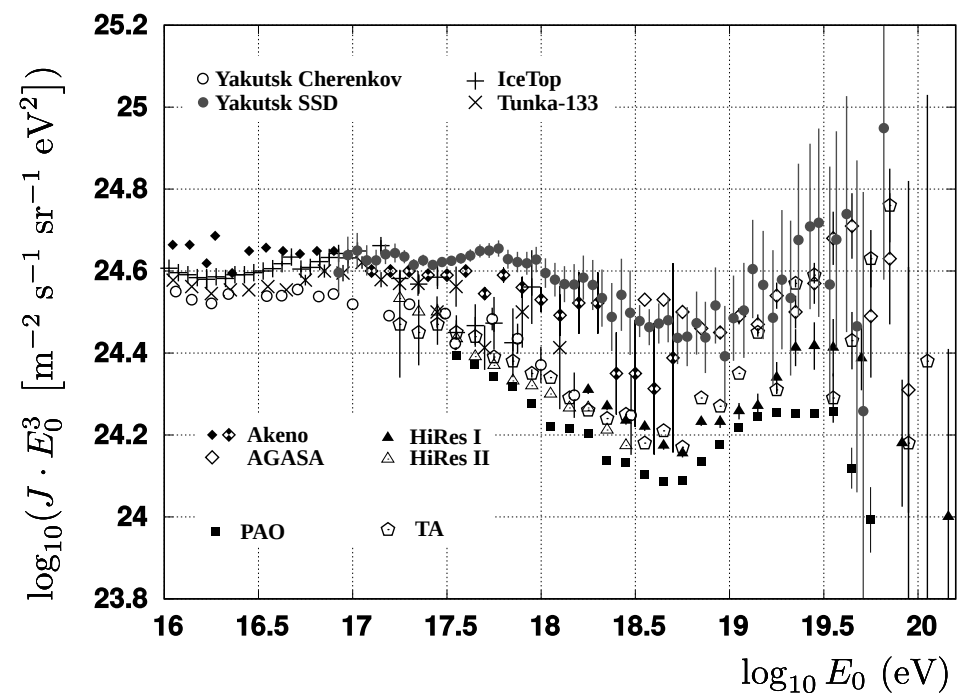

Figure 4: Differential CR energy spectrum according to the data from different world experiments.

of scintillation detectors and obtain a set of probable estimations for primary energy (2.1)-(2.4). Calculations have revealed, that in relations (1.1) and (1.4) the energy dissipated in the atmosphere in the form of electromagnetic component, was overestimated by (12-17)\% depending on the shower maximum $x_{\max }$ (see Fig. 2). This was made worse in (1.4) due to underestimation of the atmosphere transparency by $\sim 17 \%$. The new calorimetry (2.9) has lead to a lower estimated value of $E_{0}$ in comparison with (1.4) by factor $\sim 1.28$ and in significantly decreased intensity of the CR energy spectrum measured on the Yakutsk EAS array (see Fig. 4).

The work is supported by the program of Presidium of RAS "High-energy physics and neutrino astronomy" and by RFBR grant 16-29-13019 ofi-m.

\section{References}

[1] D. M. Edge, A. C. Evans, R. J. O. Garmston, H. J. Reid, A. A. Watson, J. G. Wilson and A. M. Wray, J. Phys. A: Math. Nucl. Gen. 6 (1973) 1612.

[2] A. V. Glushkov, V. M. Grigoriev, M. N. Dyakonov, T. A. Egorov, V. P. Egorova et al, Proc. of 20th ICRC, Moscow, USSR, vol. 5, pp. 494-497, Nauka, 1987.

[3] N. Sakaki, M. Chikawa, M. Fukushima, N. Hayashida, K. Honda, N. Inoue et al. Proc. of 27th ICRC, (Hamburg, Germany), vol. 1, p. 333, 2001.

[4] R. U. Abbasi, T. Abu-Zayyad, J. F. Amman, G. C. Archbold, J. A. Bellido et al. Astropart. Phys. 23 (2005) $157-174$, [arXiv: 0208301$].$

[5] V. P. Egorova, A. V. Glushkov, A. A. Ivanov, S. P. Knurenko, V. A. Kolosov et al. Nucl. Phys. B Proc. Suppl. 136 (2004) 3-11.

[6] Y. Tsunesada for TeLESCOPE ARRAY collaboration, [arXiv:1111.2507v1] (2011).

[7] R. U. Abbasi, M. Abe, T. Abu-Zayyad, M. Allen, R. Azuma et al. Astropart. Phys. 80 (2016) 131 140. 
[8] A. Aab, P. Abreu, M. Aglietta, E.J. Ahn et al. Proc. of 34th ICRC, (The Hague, The Netherlands), 2015. [arXiv:1509.03732].

[9] A. V. Glushkov and M. I. Pravdin, JETP Lett. 87 (2008) 345-348.

[10] A. V. Glushkov, Lateral distribution and total flux of Cherenkov light from EAS with primary energy $E_{0} \geq 10^{17} \mathrm{eV}$. PhD thesis, SINP MSU, Moscow, 1982. (in Russian)

[11] A. V. Glushkov, M. N. Dyakonov, T. A. Egorov, N. N. Efimov, N. N. Efremov et al. Bull. Acad. Sci. USSR: Phys. 55 (1991) 713. (in Russian)

[12] B. N. Afanasiev, B. N. Dyakonov, T. A. Egorov et al. Proc. of the Tokyo Workshop on Techniques for the Study of Extremely High Energy Cosmic Rays, pp. 35-51, 1993.

[13] A. V. Glushkov, V. P. Egorova, A. A. Ivanov, S. P. Knurenko, V. A. Kolosov et al. Proc. of the 28th ICRC, Tsukuba, Japan, vol. 1, pp. 393-396, 2003.

[14] S. I. Nikolsky, Proc. of the 5th Intern. Seminar on Cosmic Rays, La Paz, Bolivia, vol. 2, pp. 48-52, 1962.

[15] A. V. Glushkov, M. I. Pravdin and A. V. Saburov, JETP Lett. 99 (2014) 431-434.

[16] A. V. Glushkov, M. I. Pravdin and A. Sabourov, Phys. Rev. D 90 (Jul, 2014) 012005.

[17] D. Heck, J. Knapp, J. N. Capdevielle, G. Schatz and T. Thouw, FZKA 6019, Forschungszentrum Karlsruhe, 1988.

[18] N. N. Kalmykov, S. S. Ostapchenko and A. I. Pavlov, Nucl. Phys. B (Proc. Suppl.) 52 (1997) 17-28.

[19] S. Ostapchenko, Phys. Rev. D 83 (2011) 014018, [arXiv:1010.1869].

[20] E.-J. Ahn, R. Engel, T. K. Gaisser, P. Lipari and T. Stanev, Phys. Rev. D 80 (2009) 094003, [arXiv:0906.4113].

[21] T. Pierog, I. Karpenko, J. M. Katzy, E. Yatsenko and K. Werner, [arXiv:1306.0121] (2011).

[22] G. Battistoni, S. Muraro, P. R. Sala, F. Cerutti, A. Ferrari et al. Proc. of the Hadronic Shower Simulation Workshop 2006, vol. 896, pp. 31-49, 2007.

[23] A. V. Glushkov and A. V. Saburov, JETP Lett. 100 (2014) 695-698.

[24] A. V. Glushkov, Y. A. Egorov, A. A. Ivanov, S. P. Knurenko, V. P. Mokhnachevskaya, I. S. Petrov et al. this conference (2017).

[25] A. V. Glushkov and M. I. Pravdin, JETP 101 (2005) 88-97.

[26] S. P. Knurenko and A. V. Sabourov, EPJ Web of Conferences 53 (2013) 04004.

[27] M. Nagano, T. Hara, Y. Hatano, N. Hayashida, S. Kawaguchi, K. Kamata et al. J. Phys. G: Nucl. Phys. 10 (1984) 1295.

[28] M. Nagano, M. Teshima, Y. Matsubara, H. Y. Dai, T. Hara, N. Hayashida et al. J. Phys. G: Nucl. Phys. 18 (1992) 423.

[29] K. Shinozaki for AGASA collaboration, Nucl. Phys. B (Proc. Suppl.) 151 (2006) 3-10.

[30] S. F. Berezhnev, D. Besson, N. M. Budnev, A. Chiavassa, O. A. Chvalaev, O. A. Gressb et al. Nucl. Inst. Meth. A 692 (2012) 98-105, [arXiv:1201.2122v1].

[31] M. G. Aartsen, R. Abbasi, Y. Abdou, M. Ackermann, J. Adams, J. A. Aguilar et al. Phys. Rev. D 88 (2013) 042004, [arXiv:1307.3795].

[32] Z. Zundel, R. U. Abbasi, M. Abe, T. Abu-Zayyad, M. Allen, R. Azuma et al. PoS(ICRC2015)445, Proc. of 34th ICRC, (The Hague, The Netherlands), 2015. 